\title{
Geschichte für junge Frauen: Die Vermittlung historischer Bildung in Schulgeschichtsbüchern für das höhere Mädchenschulwesen
}

\author{
MARTIN NISSEN
}

\section{Geschichtsschreibung FÜR FraUeN}

Im Verlauf des 19. Jahrhunderts verlor Geschichte als Fach an deutschen Universitäten seinen minderen akademischen Rang. Aufgrund der verbesserten universitären Ausbildung stieg dementsprechend an den höheren Schulen auch das Leistungsniveau im Geschichtsunterricht. Neben dem Religionsunterricht und den klassischen Sprachen avancierte Geschichte an den Gymnasien nunmehr zu einem Kernfach (Rohlfes 1982: 11; Schneider 1982: 132). Die damit verbundene Aufwertung der historisch-politischen Bildung zielte vorrangig auf den Nachwuchs der höheren gesellschaftlichen Schichten; diese Kinder sollten im Erwachsenenalter möglichst eine führende Position einnehmen. Die Gewichtung der vermittelten Inhalte blieb im deutschen Kaiserreich freilich ideologisch stark umkämpft, denn die Geschichte war ins Zentrum der staatsbürgerlichen Erziehung gerückt (Schneider 1988: 54). Je höher die angestrebte gesellschaftliche Stellung, desto wichtiger wurden Inhalt und Umfang des im Geschichtsunterricht vermittelten Stoffes.

Für die im Unterricht vermittelten Inhalte wurden die Ergebnisse der akademischen Geschichtsschreibung maßgeblich; sie wurde in den Schul- und Lehrbüchern aufgegriffen. Im internationalen Vergleich entwickelte sich die Geschichtswissenschaft in Deutschland im 19. Jahrhundert zu einem deutlich männlich konnotierten Arbeitsfeld (Smith 1998). Durch die Institutionalisierung, Professionalisierung und fortschreitende Spezialisierung des Faches blieben 
Frauen als Autorinnen und Leserinnen weithin ausgeschlossen. Dieser Prozess wurde insofern verstärkt, als die private Erinnerung zurückgedrängt und alternative Darstellungsformen ausgegrenzt wurden. Dies wurde zunehmend mit der Vorstellung einer männlich geprägten wissenschaftlichen Objektivität verbunden. Die meisten Fachwissenschaftler an den Universitäten unterstützten die Vermittlung historischer Bildung an ein weibliches Publikum nicht. So sah Friedrich Carl von Savigny in den von Friedrich von Raumer ab 1841 in Berlin im Verein für wissenschaftliche Vorträge geplanten Vorlesungen vor Mädchen und jungen Frauen eine Herabwürdigung der Wissenschaft. Auch Leopold von Ranke trat dem Unternehmen trotz der Bitten Raumers nicht bei (Nissen 2009: 128).

Vor diesem Hintergrund könnte man annehmen, dass sich Autorinnen selbst mit historischen Arbeiten an ein weibliches Lesepublikum wandten. Zwei Tendenzen standen dieser Entwicklung jedoch entgegen: Zum einen blieb die Zahl von Historikerinnen in Deutschland im Vergleich zu Frankreich und Großbritannien marginal. Während zu Beginn des 19. Jahrhunderts - zumindest einer breiten Definition von Geschichtsschreibung folgend - Autorinnen wie Johanna Schopenhauer und Therese Huber einen weiteren Leserkreis erreichten (Epple 2003; Epple 20011), nahm die Zahl von Historikerinnen aufgrund der fortschreitenden Professionalisierung des Faches sowie der Konkurrenz durch den belletristischen Buchmarkt in der zweiten Jahrhunderthälfte ab. Louise von Kobell, Fanny Arndt, Caroline Friederike von Berg und Louise Büchner sind als Ausnahmen zu bezeichnen (Nissen 2009: 72). Zudem vermieden es die Autorinnen, ihre Position durch die Adressierung eines weiblichen Lesepublikums weiter zu schwächen - ein Hinweis hierfür ist die verbreitete Tradition der anonymen Autorenschaft (Smith 1998: 164f., Epple 2011: 27). Ähnlich wie bei populären Formen der Geschichtsschreibung diente auch in ihren historischen Darstellungen der Verweis auf führende Vertreter des Faches vielmehr dazu, die eigene Autorität abzusichern. Ein Umbruch erfolgte hier erst ab den 1890er Jahren, als Vertreterinnen der Frauenbewegung wie Lina Morgenstern, Elise Oelsner, Lily Braun und Käthe Schirmacher frauengeschichtliche Themen aufgriffen und erste professionelle Historikerinnen wie Ricarda Huch und Ermentrude von Ranke sich im Feld der akademischen Geschichtsschreibung positionierten (Paletschek 2006).

Dabei betraf der Erfolg der Bildungsgeschichte im 19. Jahrhundert Männer und Frauen gleichermaßen. Beide Geschlechter profitierten von der Schulpflicht, die seit dem frühen 19. Jahrhundert in allen deutschen Staaten bestand und zu einem Rückgang der Analphabetenquote führte. Während die Zeitungs- und Zeitschriftenlektüre vorwiegend Männern vorbehalten blieb, waren Frauen seit 
dem späten 18. Jahrhundert die Hauptadressatinnen des wachsenden Marktes für belletristische Literatur (Jäger 1980: 15). Die zunehmende Verstädterung, die Entstehung von Bildungsinstitutionen sowie die Herausbildung der bürgerlichen Kleinfamilie mit einer verstärkten Innerlichkeit des individuellen Erfahrungsraums trugen zur Entstehung eines kulturräsonierenden Publikums bei (Langenbucher 1971: 57). Während diese Entwicklung zunächst nur die höheren bildungs- und wirtschaftsbürgerlichen Schichten - maximal 5 Prozent der Bevölkerung - betraf, führten der Anstieg der Reallöhne und die sinkenden Arbeitszeiten gegen Ende des Jahrhunderts dazu, dass auch die unteren und mittleren Gesellschaftsschichten von den verbesserten Bildungschancen profitierten (Wittmann 1999: 295).

Die Ausweitung und Ausdifferenzierung des Lesepublikums spiegelt sich in der Geschichte der Geschichtsschreibung wider. Während bis zur Reichsgründung die führenden Fachwissenschaftler eine homogene deutsche Nation als Lesepublikum imaginierten, setzte ab den 1880er Jahren eine verstärkte Spezialisierung der fachwissenschaftlichen Forschung ein. Dies führte erstens zur Gründung spezieller Fachzeitschriften, zweitens zur zunehmenden Ausgrenzung abweichender Darstellungsformen, drittens zu einem Boom von der Fachwissenschaft vernachlässigter, aber in einer breiteren Öffentlichkeit beliebter Themen wie Regional-, Welt- und Kulturgeschichte sowie viertens zur verstärkten Produktion von Geschichtsliteratur, die sich an spezielle Lesepublika wie Frauen, Arbeiter und Kinder richtete.

Die Zahl der historischen Werke, die sich vorrangig auf ein weibliches Publikum bezogen, blieb jedoch begrenzt. Ersten Ergebnissen zufolge lassen sich folgende Typen unterscheiden: Erstens historische Beiträge in Frauenzeitschriften wie der ab 1838 von der Publizistin und Übersetzerin Louise Marezoll in Leipzig herausgegebenen Frauenzeitung (1840-44 unter dem Titel Frauenspie$\mathrm{gel}$ ), in der neben Novellen, Fortsetzungsromanen, Gedichten, Andachten und Gebeten auch biographische sowie lokal- und kulturgeschichtliche Beiträge publiziert wurden (Weckel 1998: 32f.); zweitens Sammlungen von Vorträgen wie der Vorlesungszyklus des Leipziger Kulturhistorikers Karl Friedrich Biedermann von 1854/55, der ab 1855 in der Gartenlaube veröffentlicht wurde und 1856 in Buchform unter dem Titel Frauen-Brevier erschien (Biedermann 1856); drittens schließlich Schulgeschichtsbücher für höhere Mädchenschulen, die sich bis zur Neuordnung des Mädchenschulwesens in den 1890er Jahren als Schul- und Hausbücher auch an ein breiteres Lesepublikum richteten.

Der Schwerpunkt der folgenden Untersuchung liegt auf den Geschichtsbüchern für das höhere Mädchenschulwesen, bei denen der Bezug auf ein weibliches Lesepublikum bereits institutionell vorgegeben ist. Wie genauer zu zeigen 
sein wird, haben Schulgeschichtsbücher eine weite Verbreitung über häufig lange Zeiträume hinweg gefunden. Wo sind - im Vergleich zu den Schulgeschichtsbüchern für Knabenschulen - die Besonderheiten in den Darstellungsformen zu sehen? Welche didaktischen Konzepte lagen den Werken zugrunde? Welche Geschlechterbilder wurden anhand historischer Beispiele vermittelt und was waren bevorzugte Themen? Die Untersuchung legt methodisch den Schwerpunkt auf paratextuelle Elemente, also auf Vorworte und Einleitungen zu Schulgeschichtsbüchern für das höhere Schulwesen, die 2011 von Wolfgang Jacobmeyer in dem dreibändigen Werk Das deutsche Schulgeschichtsbuch 1700-1945 herausgegeben wurden (Jacobmeyer 2011).

\section{DAS DEUTSCHE SCHULGESCHICHTSBUCH FÜR HÖHERE MÄDCHENSCHULEN}

Im Vergleich zu anderen Formen der Geschichtsschreibung sind Schulgeschichtsbücher als hybride Publikationen zu bezeichnen, die einerseits ein normiertes staatliches Genehmigungsverfahren durchlaufen, sich zugleich jedoch an ein breiteres Lesepublikum auch außerhalb des schulischen Kontextes wenden. Die Lektüre erfolgt nicht freiwillig, sondern verpflichtend im Rahmen staatlicher Kontrolle. In seiner didaktischen Bedeutung fällt das Schulgeschichtsbuch zwar hinter den Lehrervortrag zurück. Aufgrund der vorgegebenen inhaltlichen Nähe zwischen Schulbuch und mündlichem Unterricht kann dennoch von einer hohen Bedeutung bei eindeutiger Adressierung ausgegangen werden. Wolfgang Jacobmeyer zufolge ist das Schulgeschichtsbuch »unstrittig das am weitesten verbreitete Medium moderner Gesellschaften zur Überlieferung von Geschichte« (Jacobmeyer 2011: 10).

Für Mädchen gab es seit dem 18. Jahrhundert in allen deutschen Staaten gesonderte Schulen. Die eingeführte Schulpflicht machte die Gründung eigener Mädchenschulen erforderlich. Für die Töchter aus den höheren gesellschaftlichen Schichten verbot sich der Besuch der Elementarschulen aus Gründen der sozialen Distinktion. Bis zu Beginn des 20. Jahrhunderts blieb ihnen wiederum der Besuch des Gymnasiums verwehrt. Gegründet wurden die höheren Mädchenschulen vielfach als Abteilungen von Knabenschulen. Zunehmend bildeten sich jedoch eigenständige, auch räumlich getrennte Schulen heraus. Im Gegensatz zu den Knabenschulen fielen die Mädchenschulen nicht unter staatliche Trägerschaft, sondern gingen auf private Initiativen zurück. Die Schülerinnen stammten aus den gebildeten und vermögenden städtischen Gesellschaftsschichten, die für den Schulbesuch ihrer Töchter ein hohes Schulgeld zahlten. Die 
Schulzeit umfasste im Anschluss an eine vierjährige Elementarschulzeit neun, häufig zehn Unterrichtsjahre, womit die Gymnasialschulzeit um ein Jahr übertroffen wurde. Aufgrund des verwehrten Zugangs für Frauen zu höherer Bildung führten an den höheren Mädchenschulen überwiegend Lehrer den Unterricht durch. In den katholisch geprägten Staaten kamen Ordensschwestern als Teil des Lehrpersonals hinzu (Kraul 1991; Küpper 1991).

Geschichte war seit Beginn des 18. Jahrhunderts als ordentliches Lehrfach an allen höheren Schulen anerkannt, wobei die katholischen Länder den protestantischen nachfolgten (Rohlfes 1982: 35). Im Verlauf des 19. Jahrhunderts löste sich Geschichte als Fach zunehmend vom Religionsunterricht und der Morallehre. Vor allem in den unteren Klassen der höheren Schulen wurde der Schwerpunkt auf Kirchengeschichte und Alte Geschichte gelegt. Ab der Mitte des Jahrhunderts nahm die Neue Geschichte mit besonderer Betonung der jeweiligen Regional- und Landesgeschichte einen Aufschwung. An den Elementar- und Volksschulen hingegen war die Bedeutung der Vermittlung historischer Bildung gering. Bis zur Mitte des Jahrhunderts gab es keinen eigenständigen Realienunterricht, zu dem auch der Geschichtsunterricht gehört hätte. Geschichte war an den höheren Mädchenschulen zwar nicht Hauptfach wie weibliches Arbeiten, Deutsch, Religion und Fremdsprachenunterricht, doch gehörte es bei steigender Stundenzahl überall zum Kernbestand (Küpper 1987: 182f.). Um 1900 lag der Unterrichtsanteil zusammen mit Kunstgeschichte und Erdkunde rund eineinhalb Mal höher als an den Knabenschulen (Kraul 1991: 291). Mit zwei bis maximal drei Wochenstunden blieb der Anteil an der gesamten Unterrichtszeit jedoch moderat (Schneider 1997: 495-509).

Nachdem sich Geschichte als Fach im Fächerkanon der höheren Schulen etabliert hatte, bildete sich das Schulgeschichtsbuch seit dem frühen 19. Jahrhundert als eigenständige Gattung heraus. 1857 verfügte die Kultusbehörde in Preußen, dass der Geschichtsunterricht auf der Grundlage eines Lehrbuchs zu erfolgen habe (Erdmann 1982: 78). Diese Vorschrift führte zu einem starken Anstieg der Produktion von Schulgeschichtsbüchern ab den 1860er Jahren, die in der wilhelminischen Phase des Kaiserreichs ihren Höhepunkt erreichte. Die Zeit des wilhelminischen Kaiserreichs kann dabei als das »Zentrum der Gattungsgeschichte« des Schulgeschichtsbuchs bezeichnet werden (Jacobmeyer 2011: 177). In dem Zeitraum zwischen 1890 und 1918 weist Wolfgang Jacobmeyer die Produktion von 478 neuen Schulgeschichtsbüchern nach. Damit sind in diesen 29 Jahren ebenso viele Lehrbücher erschienen wie in den rund 150 Jahren zwischen 1700 und 1850 zuvor. In den anschließenden Phasen während der Weimarer Republik und im Dritten Reich ging die Produktion neuer Schulgeschichtsbücher 
trotz stark veränderter politischer Rahmenbedingungen quantitativ wieder zurück.

Für den Zeitraum zwischen 1890 und 1918 liegen auch für die Schulgeschichtsbücher für höhere Mädchenschulen die genauesten Zahlen vor (Jacobmeyer 2011: 177f.). Von den ermittelten 478 Titeln lassen sich 80 Prozent spezifischen Schulformen zuordnen. Dabei entfallen 44 Titel auf Mädchenschulen, 120 auf Gymnasien, 141 auf Volks- und 78 auf Mittelschulen. Unter den 44 Schulgeschichtsbüchern für Mädchenschulen ist der größte Teil, nämlich 41 Titel, dem Typus des narrativen Lehrbuchs zuzuordnen, dem zwei Lehrbücher für Lehrer und ein Lernhilfsbuch entgegenstehen. Der inhaltlich anspruchsvollste Typus des Quellen-Lehrbuchs, der bei den Gymnasiallehrbüchern zwölf von 120 Titeln ausmacht, fehlt vollständig. Die größten Unterschiede im Vergleich zu den Schulgeschichtsbüchern für Jungen bestehen für Gymnasien, wohingegen die Unterschiede zu den Schulgeschichtsbüchern der Volks- und Mittelschulen geringer ausfallen. Im Gegensatz zum 20. Jahrhundert sind Schulgeschichtsbücher im 19. Jahrhundert keine von Verlagen initiierten Projekte, an denen eine Vielzahl von Akteuren beteiligt ist. Noch überwiegt der Typus des Einzelautors, der meist über eine langjährige Unterrichtserfahrung an Mädchenschulen verfügt. Es bestehen nur geringe Unterschiede zwischen den Autoren von Schulgeschichtsbüchern für Knaben- und denen für Mädchenschulen. Fast ausschließlich sind die Autoren selbst als Lehrer tätig. Sie sind überwiegend evangelisch und verfügen über eine akademische, fachlich einschlägige Ausbildung, die häufig mit der Promotion abgeschlossen wurde. 44 Prozent sind Lehrer und Direktoren der Lehrerinnenseminare, 31 Prozent Gymnasiallehrer und lediglich 12 Prozent Lehrer an Realschulen oder Vertreter der Schulverwaltung (Jacobmeyer 2011: 193). Lehrerinnen oder Schriftstellerinnen als Verfasserinnen von Schul- und Lehrbüchern für höhere Mädchenschulen sind die Ausnahme. Trotz des akademischen Hintergrundes der Autoren wurden in den Darstellungen kaum eigene Forschungsleistungen verarbeitet, sondern überwiegend Bekanntes aus Lehrund Überblickswerken zusammengefasst. Im späten 19. Jahrhundert nahmen Unternehmungen, an denen mehrere Verfasser beteiligt waren, zu. Das Bildungsniveau stieg weiter an, wobei noch immer die praktische Erfahrung in der Lehrtätigkeit Voraussetzung für eine erfolgreiche Autorenschaft war.

Unter den wenigen Autorinnen von Mädchenschulbüchern überwog die Position der gemäßigten bürgerlichen Frauenbewegung, die sich 1865 im Allgemeinen Deutschen Frauenverein zusammengeschlossen hatte (Kraul 1991: 280). Auf der Basis der bestehenden Geschlechterunterschiede forderten sie gleiche Bildungschancen für das weibliche Geschlecht. Ihr Engagement galt der Förderung weiblicher Bildung und Erwerbstätigkeit, wobei den Besonderheiten der 
Frau und der Vermittlung weiblicher Tugenden Rechnung getragen werden müsse. Weiterführende Forderungen nach Zugang zu höherer Bildung als Voraussetzung für akademische Berufswege wurden erst ab den 1890er Jahren verstärkt geäußert. Eine typische Vertreterin der gemäßigten emanzipatorischen Position ist die Schriftstellerin Ella Mensch, die im Vorwort zu ihrem Leitfaden für den Unterricht in der Weltgeschichte an höheren Mädchenschulen von 1910 forderte:

»Um schließlich die einseitige Auffassung zu tilgen, daß die Weltgeschichte lediglich eine Geschichte der Männer sei, muß schon im >Leitfaden< das Wirken und Schaffen der Frau, da, wo es als Einschlag im großen Gewebe zu erkennen ist, sichtbarlich, mit wenigen aber deutlichen Strichen hervorgehoben werden, ganz besonders in der deutschen Geschichte, wo sich an den wichtigen Wendepunkten unseres historischen Lebens auch stets der kulturelle Einfluß der Frauen geltend macht, mögen sie nun wie die Schwabenherzogin Hadwig oder die Kaiserinnen aus sächsischem Hause die Verantwortung für ein ganzes Gemeinwesen tragen - oder wie im Jahr der Befreiungskriege von 1813 als Gesamtheit die guten und gesunden Regungen im Volksgeist unterstützen.« (Jacobmeyer 2011: 1324)

Die Gattungsgeschichte des Schulgeschichtsbuchs für höhere Mädchenschulen umfasst den Zeitraum von Beginn des 19. bis zu Beginn des 20. Jahrhunderts. Eine verstärkte Produktion lässt sich erst seit den 1820er Jahren feststellen, wobei zwischen 1850 und 1890 eine geringe Zahl an Titeln den Markt beherrschte. Der wichtigste Einschnitt waren die am 31. Mai 1894 vom preußischen Kultusministerium erlassenen Bestimmungen über das Mädchenschulwesen, die Lehrerinnenbildung und die Lehrerinnenprüfungen, in denen zum ersten Mal die Verwendung eines Schulgeschichtsbuchs an Mädchenschulen verbindlich vorgeschrieben wurde (Ministerium 1894: §7 u. 8). In der Folge erschienen in dem kurzen Zeitraum bis 1900 zwölf Schulgeschichtsbücher, die sich wie Ferdinand Roßbachs Leitfaden für den Unterricht in der deutschen Geschichte in den oberen Klassen höherer Mädchenschulen von 1895 explizit auf die Bestimmungen über das Mädchenschulwesen bezogen. Die Verlage setzten dabei den Erlass nicht lediglich um, sondern nutzten die Vorgaben auch als Werbemittel zur Steigerung des eigenen Absatzes.

Die verstärkte Produktion in den späten 1890er Jahren ist jedoch nur als Vorbote für das bevorstehende Ende der Gattung zu verstehen. An den neuen Schulbüchern, die explizit im Hinblick auf ein weibliches Lesepublikum hin geschrieben wurden, entzündete sich die Kritik an der geschlechtsspezifischen Einteilung der vermittelten Unterrichtsinhalte (Jacobmeyer 2011: 196f.). So forderte etwa Friedrich Neubauer, einflussreicher Direktor des Frankfurter Lessing-Gym- 
nasiums, ein Ende der didaktischen Ungleichbehandlung. Die Forderungen nach einer Angleichung des Leistungsniveaus an sämtlichen höheren Schulen fand Ausdruck in dem ministerialen Erlass vom 15. August 1908, in dem die Unterschiede zwischen Knaben- und Mädchenunterricht zurückgenommen wurden. Der Unterricht an Mädchenschulen sollte nun in allen deutschen Staaten den Zugang zu höherer akademischer Bildung und zu den Universitäten ermöglichen, die für Frauen je nach Staat offiziell zwischen 1900 und 1909 - zuerst in Baden, zuletzt in Mecklenburg - geöffnet wurden. Auch wenn die Vorbehalte gegenüber gelehrten Frauen nach 1900 bestehen blieben, war die Richtung vorgegeben. Eine gesonderte Darstellung der Geschichte für Mädchen und junge Frauen wurde zunehmend als gegenstandslos erachtet.

\section{DidakTISCHE KONZEPTE IM GESCHICHTSUNTERRICHT}

In seiner didaktischen Bedeutung trat das Schulbuch zwar hinter den Lehrervortrag zurück. So galt der Lehrervortrag insbesondere bei den didaktischen Zielen Motivation und Wertevermittlung weiterhin als Maß aller Dinge (Jacobmeyer 2011: 150f.). Dennoch ist die Bedeutung des Schulbuchs als sekundäres Unterrichtsmittel hoch einzuschätzen. Es diente als wichtiges Instrument zur Umsetzung der ministerialen Vorgaben und als zentrale Form der Qualitätssicherung im Unterricht.

Die Zahl an Vorworten und Einleitungen zu Schulgeschichtsbüchern für Mädchenschulen, in denen didaktische Konzepte erläutert wurden, blieb im 19. Jahrhundert begrenzt. Hierbei sind vor allem die Schulgeschichtsbücher der ersten Hälfte des 19. Jahrhunderts interessant. Eine spezielle Darstellung der Geschichte für Mädchen und junge Frauen war neu und insofern besonders erklärungsbedürftig. Ausführlich äußerte sich Friedrich Nösselt (1781-1850), einer der produktivsten und einflussreichsten deutschsprachigen Autoren von Mädchenschulbüchern im 19. Jahrhundert. Nösselt hatte in Halle Theologie studiert und war seit 1804 in Berlin, seit 1809 in Breslau als Gymnasiallehrer tätig. Im selben Jahr gründete er in Breslau eine Töchterschule, an die 1836 ein Seminar für künftige Erzieherinnen angeschlossen wurde. Parallel zu seiner pädagogischen Tätigkeit verfasste Nösselt zahlreiche historische, geographische und literaturgeschichtliche Lehrbücher, die bis zur Mitte des 19. Jahrhunderts weite Verbreitung fanden. Nösselts didaktisches Konzept bei der Vermittlung historischer Bildung, mit der er sich an »heranwachsende Mädchen der gebildeten Stände« wandte (Nösselt 1822: VII), beruhte auf der Annahme, dass die Geschlechter über unterschiedliche Veranlagungen verfügten, die ihnen unter- 
schiedliche gesellschaftliche Aufgaben zuwiesen. Ausführlich begründete er dies im Vorwort zu seinem Lehrbuch der Weltgeschichte für Töchterschulen und zum Privatunterricht heranwachsender Töchter von 1822, das bis 186714 Auflagen erfuhr. In Inhalt und Form müsse der Geschichtsunterricht die spezifischen Erfordernisse weiblicher Bildung berücksichtigen:

»Es braucht hier nicht erst bewiesen zu werden, daß die Geschichte den Mädchen ganz anders vorgetragen werden müsse, als den Knaben und Jünglingen. Wenn diese nicht nur einen allgemeinen Ueberblicke über die ganze Geschichte, sondern auch eine in die einzelnen Theile derselben eingehende Kenntniß nöthig haben und die einzelnen Völker Schritt vor Schritt verfolgen müssen, mit beständiger Berücksichtigung der Chronologie, so ist dies Alles für Mädchen unnütz, für die es hinlänglich ist, wenn sie die Hauptbegebenheiten und diejenigen Thatsachen lernen, welche das weibliche Gemüth besonders ansprechen, an denen sie die Schönheit der Seelengröße und die Verwerflichkeit des Lasters und der Schwäche kennen lernen können, und welche ganz vorzüglich eine väterliche Weltregierung beweisen. Ueberhaupt scheint dem Verfasser, daß für Mädchen die Geschichte von der gemüthlichen Seite dargestellt werden müsse. Vieles aus ihr, was Knaben lernen, müssen die Mädchen auch wissen; aber Unzähliges muß beim weiblichen Unterrichte ganz weggelassen werden; dagegen sind viele Thatsachen für das weibliche Herz äußerst ansprechend, die man doch Knaben nicht vorzutragen pflegt, um für Wichtigeres Zeit zu behalten.«(Nösselt 1836, Bd. 1: VII)

Mit der Betonung des biographischen Elements und der Nähe der Erzählung zu den historischen Quellen werden Darstellungsformen gewählt, die auch als Mittel populärer Geschichtsschreibung bekannt sind. Die Erzählung wiederum, die sich im Sinne der didaktischen Reduktion auf die wichtigsten Ereignisse konzentrieren müsse - »Nichts tödtet den historischen Sinn mehr als die compediarische oder tabellarische Methode« (Nösselt 1836, Bd. 1: V) - hatte sich auch in der Fachwissenschaft im 19. Jahrhundert als wichtigste Darstellungsform durchgesetzt. Abweichungen in der Darstellung lassen sich jedoch in der notwendigerweise stärkeren Betonung des »Gemüths«, der Frömmigkeit und der Moral in Mädchenschulbüchern feststellen. So solle der Vortrag »lebhaft, kindlich und gemüthlich « sein ohne dabei die moralische Bewertung zu vernachlässigen (Nösselt 1836: Bd. 1: V). In seinem Lehrbuch der Geschichte der Deutschen für höhere Töchterschulen und die Gebildeten des weiblichen Geschlechts von 1828 fasst Nösselt den »Hauptzweck« des Geschichtsunterrichts folgendermaßen zusammen: 
»[...] das moralische und religiöse Gefühl zu beleben und die Ueberzeugung recht fest in die jugendlichen Herzen zu prägen, daß alles, was da geschieht und von je her geschehen ist, unter der allweisen Leitung der göttlichen Vorsehung stehe, und daß ohne Ausnahme aus guten Handlungen Glück und Segen, aus schlechten nichts als Unheil und Unsegen hervorgehen müsse. Was hilft es den Mädchen, wenn sie noch so viele Namen, Begebenheiten und Jahreszahlen wissen, und diese große Lehre, die auf allen Blättern der Weltgeschichte so laut und deutlich gepredigt wird, nicht begriffen haben!« (Jacobmeyer 2011: 574).

Insgesamt blieb die didaktische Tradition des Geschichtsunterrichts als Schule des Lebens (Historia magistra vitae), die in der akademischen Geschichtsschreibung zurückgenommen und in den Schulgeschichtsbüchern für Knabenschulen zumindest abgeschwächt wurde, an Mädchenschulen länger bestehen. Die Schulung des historischen Urteils wurde als wichtiger erachtet als die Vermittlung reinen Faktenwissens. Die Schülerinnen dürfe man Nösselt zufolge »nicht mit Jahreszahlen überhäufen, sie nicht die Reihen der Königsnamen auswendig lernen lassen« (Nösselt 1836, Bd. 1: V). Es müsse alles vermieden werden, »was einem Mädchen den Anstrich von gelehrter Bildung giebt.«Hervorzuheben seien vielmehr »gute und böse Beispiele, folgenreiche Thatsachen, besonders Handlungen merkwürdiger Frauen« (Nösselt 1836, Bd. 1: V). Bei der Darstellung selbst wird das Verhalten historischer Protagonistinnen in moralischen Kategorien gefasst, denen spezifische geschlechtsanthropologische Rollenmuster zugrunde gelegt werden. So sind - um ein Beispiel zu geben - bei der Abhandlung von Luthers Biographie die Rollen der Eltern so gestaltet, dass dem Vater der vernünftige Part zufällt, während die Mutter weiblich konnotierte Tugenden verkörpert:

»Auch seine Mutter, eine tugendsame und gottesfürchtige Frau, hatte einen segensreichen Einfluß auf ihres Sohnes Erziehung. Von ihr erhielt er besonders den Sinn für stille, häusliche Frömmigkeit, und es ist der große Segen nicht zu berechnen, den fromme Mütter über die Gemüther ihrer Kinder verbreiten.«(Nösselt 1836, Bd. 3: 2)

Noch stärker betont wird die Bedeutung der Geschlechteranthropologie für die Konzeption der Geschichtsvermittlung in der zweibändigen Weltgeschichte für Töchterschulen und zum Privatunterricht des Preßburger Pädagogen Tobias Gottfried Schröer (1791-1850) von 1841, die dieser unter dem Pseudonym Chr. Oeser veröffentlichte. Schröer hatte 1816-17 in Halle zwei Semester Theologie studiert und war aus finanziellen Gründen vorzeitig in seine Heimatstadt Preßburg zurückgekehrt, wo er ab 1817 am evangelischen Lyzeum Latein, Grie- 
chisch, Deutsch, Geographie, Geschichte und Ästhetik unterrichtete. 1818 verfasste er einen Organisationsentwurf für eine neu zu gründende Töchterschule, deren Leitung er bis 1824 innehatte. 1824 wurde Schröer an dem Lyzeum Subrektor, 1838 Professor für die höheren Klassen. Politisch gehörte er unter den Ungarndeutschen zur gemäßigten Richtung und unterstützte im Vormärz die liberale Bewegung. In dem Vorwort zu seiner Weltgeschichte für Töchterschulen und zum Privatunterricht betont Schröer in ähnlicher Weise wie Friedrich Nösselt die Bedeutung der Herzensbildung im Geschichtsunterricht. Dieser diene bei den jungen Frauen nicht der Berufsvorbereitung, sondern vorrangig der Vermittlung von Moral auf religiös-sittlicher Grundlage:

»Denn für diese [Töchter] hat die Geschichte meist nur formellen Nutzen, und sie lernen sie nicht etwa, um von ihr im bürgerlichen oder wissenschaftlichen Leben wie Staatsbeamte oder Lehrer Gebrauch zu machen, sondern einzig, um Geist und Herz zu bilden. Unsere Jungfrauen sollen nämlich Geschichte lernen, damit sie den Ernst des Lebens und die Hand Gottes in den Schicksalen einzelner Menschen und ganzer Völker wahrnehmen, um sich zu hüten, jenen Geschöpfen zu gleichen, die noch von der frivolen Leichtfertigkeit befallen sind, welche durch französische Sitten und Gouvernanten in unser Vaterland gebracht worden sind; [...] Unsere Töchter sollen Geschichte lernen, damit sie die Aufgabe unserer Nation, als Lehrmeisterin und Gesetzgeberin des Erdkreises und Verbreiterin ächter Humanität im häuslichen, wie im bürgerlichen Leben erkennen, und, nach Vorbilde alter deutscher Frauen, häuslich, treu und ehrbar leben. [...] Unsere Frauen sollen Geschichte wissen, damit sie erfahren, wie Kunst und Wissenschaft, Tugend und Glaube in Zeiten der Rohheit und Ausartung bei ihnen, und nur bei ihnen, eine Freistätte gefunden, wie aber auch lasterhafte Weiber den Untergang ganzer Völker beschleunigt haben.«(Oeser 1843, Teil 1: Vf.)

Schröer gliedert die Darstellung in die Epochen Altertum, Mittelalter und Neuzeit, wobei er an das Ende der Epochenbeschreibung je ein Kapitel zur Situation der Frauen in der jeweiligen Zeit anhängt. Die Geschichte der Frauen dient dabei als Spiegel der jeweiligen historischen Zustände. Der Zustand des weiblichen Geschlechts kontrastiert bzw. verstärkt den welthistorischen Entwicklungsgang, ohne ihn jedoch maßgeblich zu beeinflussen. So heißt es zur Einführung der Geschichte der Frauen im Reformationszeitalter:

»In einem Zeitalter, wo Kunst und Wissenschaft neuerwacht zum Leben bei den meisten Völkern die Sitten milderten, mußte sich auch das Loos des weiblichen Geschlechts freundlicher gestalten.«(Oeser 1843, Teil 3: 79) 
Die aufeinander folgenden Epochen werden in der Darstellung einem stark moralisierenden Urteil unterzogen und in kontrastierender Weise bewertet, wobei der Anteil der Frauen bei der Abfolge von Sittlichkeit und Sittenverfall verstärkend wirkt. So folgte Schröer zufolge auf die Blüte des klassischen Altertums die römische Dekadenz der Spätantike, auf die Hochkultur des Hochmittelalters der Sittenverfall des Spätmittelalters, auf die Läuterung im Reformationszeitalter die Unzucht der höfischen Kultur, die erst durch die Tugendhaftigkeit des bürgerlichen Zeitalters abgelöst wurde. In der Darstellung dienen die christliche Kultur, das Deutschtum und der evangelische Glaube als Quellen der Sittlichkeit. Quellen des Sittenverfalls sind die römische Dekadenz, die Korruption in der spätmittelalterlichen Kirche sowie die Galanterie der französischen Hofkultur. Zeitgenössische bürgerliche Idealvorstellungen werden dabei - wie bei vielen historiographischen Darstellungen des 19. Jahrhunderts üblich - auf vergangene Zeiten übertragen. So sei durch die Verrohung der Sitten im späten Mittelalter das »stille häusliche Glück im freundlichen Zusammenleben liebender Ehegatten« verloren gegangen, genauso »bei manchem Weibe [die] häusliche Betriebsamkeit und andere weibliche Tugenden «(Oeser 1843, Teil 2: 310).

Der christlich-moralische Impetus fällt in Schröers Werken besonders deutlich aus. So heißt es auch in dem Vorwort zu dem Kurze[n] Leitfaden der allgemeinen Weltgeschichte für Töchterschulen und zum Privatunterrichte für das weibliche Geschlecht von 1842, den er zusätzlich zu seiner dreiteiligen Weltgeschichte für Töchterschulen verfasste:

»Und nun, liebe Mädchen, sei euer leibliches und geistiges Erblühen in voller Gesundheit und Reinheit empfohlen Gott dem Vater, damit ihr recht wackere und herzensgute Frauen dereinst werdet, Frauen, wie sie der Deutsche gern hat und liebt und ehrt.« (Jacobmeyer 2011: 700)

Zwar wird die Vermittlung weiblicher Tugenden in den Vorworten zu Schulgeschichtsbüchern bis zum späten 19. Jahrhundert weiterhin als zentrales Unterrichtsziel benannt, doch werden die Begründungen weniger christlich-moralisch, sondern zunehmend patriotisch-national ausgerichtet. Die Ablösung von einer vorrangig christlich-moralisch geprägten Pädagogik zugunsten einer patriotischnationalen Wertevermittlung zeichnet sich in dem Lehrbuch Weltgeschichte für höhere Töchterschulen des Berliner Pädagogen Carl Wernicke von 1849 ab, bei dem es sich um das in den Jahrzehnten zwischen 1850 und 1890 wichtigste deutsche Schulgeschichtsbuch an höheren Mädchenschulen handelt. Bis 1909 wurde Wernickes Lehrbuch im Berliner Verlag Nauck bzw. Nauck'sche Erben $34 \mathrm{Mal}$ aufgelegt. Erst durch den Boom an Schulgeschichtsbüchern in der Folge des 
preußischen Erlasses zur Neuordnung des höheren Mädchenschulwesens vom 31. Mai 1894 verlor das Werk seine Vorrangstellung.

In dem Vorwort wird die Auseinandersetzung mit Forderungen nach höherer Mädchenbildung zur Mitte des 19. Jahrhunderts deutlich. Wernickes Pädagogik beruht auf der gängigen Sonderanthropologie für Mädchen und junge Frauen, die den Ausgangspunkt für die Forderung nach unterschiedlichen, geschlechtsspezifischen Darstellungsformen begründet. Von Natur aus unterscheide sich die Wahrnehmung von Jungen und Mädchen grundlegend, wonach das Interesse des Jungen auf das Allgemeine, das des Mädchens auf das Spezielle abziele. Die Jungen seien befähigt, die Dinge in ihrer Gesamtheit wahrzunehmen, wohingegen die Weltsicht des Mädchens im Detail verharre. Das Mädchen erfasse die Welt über das Gefühl, der Junge über den Verstand. Für die Darstellung der Geschichte ergäben sich Wernicke zufolge grundlegende Konsequenzen:

»Nirgends dürfen daher dem Mädchen nur allgemeine Umrisse gegeben werden. Der Lehrer darf ihm den Entwicklungsgang des großen Drama's der Weltgeschichte nicht nur in großen Zügen zeichnen. In lebendigen Gestalten muß er die Persönlichkeiten, in denen sich die Entwicklung des Menschengeschlechts in irgend einer Zeit gleichsam concentrirte, dem jugendlichen Geiste vorführen, damit aus diesem concreten Bilde sich dem Kinde das Allgemeine herausbilde und selbst eine lebendige Gestalt gewinne. Ueberall muß der Lehrer das biographische Element vorherrschen lassen [...]« (Jacobmeyer 2011: 747f.)

Sämtliche, zu weit reichende Emanzipationsbestrebungen seien mit den Gesetzmäßigkeiten der Natur unvereinbar. Bestehende Forderungen nach einer Gleichstellung der Geschlechter lehnt Wernicke als »krankhafte Erscheinung unserer Zeit« ab (Jacobmeyer 2011: 747). Gleichzeitig verschließt er sich jedoch nicht Forderungen nach einer Verbesserung der höheren Mädchenschulbildung. Ausgangsbedingungen und Ziele seien der naturgegebenen Geschlechteranthropologie zufolge zwar verschieden, das Leistungsniveau in Gymnasien und höheren Mädchenschulen solle sich jedoch nicht grundsätzlich unterscheiden. Hier wird der Entwicklungsgang der höheren Mädchenbildung am Ende des 19. Jahrhunderts bereits sichtbar.

Wernickes Forderungen nach einer geschlechtsspezifischen Darstellungsform in Schulgeschichtsbüchern für das höhere Mädchenschulwesen können als stellvertretend für die didaktischen Konzepte der zweiten Hälfte des 19. Jahrhunderts angenommen werden. Im Zentrum standen dabei das biographische Prinzip, die exemplarische Erzählung, die Wernicke zufolge »heiter und freundlich« sein solle, und das moralische Beispiel, das der Vermittlung weiblicher 
Tugenden diente. Sprachlich sollte die Darstellung mit einfachem Satzbau und unter Vermeidung von Fremdwörtern und Fachtermini schlicht gestaltet sein. Im Vergleich zu den Lehrbüchern für Knabenschulen traten somit die didaktischen Formen des Schulbuchs als Hilfsmittel für die Repetition, das Tabellenwerk mit Zeittafeln und das Kompendium, eine Art Kurzabriss der Geschichte, in den Hintergrund (Jacobmeyer 2011: 127 ff.). Geschuldet war dies allerdings auch der insgesamt geringeren Investitionsquote bei Lehrmitteln für den Mädchenunterricht, die die zentrale Darstellungsform der narrativen Langfassung gänzlich in den Mittelpunkt treten ließ.

Thematisch lagen in der ersten Hälfte des 19. Jahrhunderts die Schwerpunkte auf der Alten Geschichte, der Kirchengeschichte und der antiken Mythologie, der eigene Lehrbücher gewidmet wurden (Jacobmeyer 2011: 571). Friedrich Nösselt zufolge sollte die antike Mythologie zwar nicht mehr als eine Stunde pro Woche unterrichtet werden. Didaktisch hielt er sie jedoch für besonders geeignet, die Ausprägung des weiblichen »Gemüths « zu fördern und den ästhetischen Sinn zu schulen. Im Verlauf des Jahrhunderts verloren Alte Geschichte und Mythologie zunehmend an Bedeutung. Die Schulbücher für Knaben und Mädchen näherten sich thematisch an, wobei im letzten Viertel des 19. Jahrhunderts die neuere und neueste Geschichte in den Vordergrund trat. Thematische Unterschiede bestanden darüber hinaus in der Beschränkung der politischen Geschichte auf die wesentlichen Entwicklungsgänge; Diplomatie-, Verwaltungs-, Verfassungs- und Militärgeschichte wurden weitgehend ausgespart. Zu verzichten sei Tobias Gottfried Schröer zufolge bei den Mädchen zudem auf das Auswendiglernen von Jahreszahlen, die Repetition genealogischer Reihen und die Abhandlung von Kriegsverläufen:

»Am wenigsten Interesse für sie können Kriege, Feldzüge und Schlachten haben; es ist genug, ihnen die Arbeit der Männer in einigen Gemälden zu zeigen; über ganze Zeitläufe, in denen die Menschheit mehr zerstörend als bauend zu schauen ist, kann man flüchtig hinwegeilen. Desto länger muß bei der Entwickelung menschlicher Cultur, bei Sitten und Gebräuchen, Kunst, Wissenschaft und Religion verweilt werden und am längsten bei ausgezeichneten weiblichen Charakteren.«(Oeser 1843, Teil 1: VIII)

In den Schulgeschichtsbüchern sowohl für Jungen als auch Mädchen setzt sich im Laufe des 19. Jahrhunderts die Einteilung in Altertum, Mittelalter und Neuzeit durch, wobei die Epochen zunehmend in einem Lehrbuch abgehandelt werden. Inhaltlich erweitert sich das Spektrum an der Wende vom 19. zum 20. Jahrhundert. Ein Schwerpunkt liegt nun auf Lebensbildern, insbesondere der »vaterländischen Geschichte« (Ministerium 1894: 29) sowie auf der Darstellung der 
Kulturgeschichte. Dieser müsse, wie es in Emil Brockmanns dreibändigem Lehrbuch der Geschichte für katholische höhere Mädchenschulen, LehrerinnenBildungs-Anstalten und freie Fortbildungskurse von 1905 heißt, »der weiteste Raum« gewährt werden (Jacobmeyer 2011: 1289). Bei den Biographien seien Vorbilder wie treue Gattinnen, Wohltäterinnen und glaubensstarke Christinnen herauszustellen, so der Leipziger Schuldirektor Louis Mittenzwey (Jacobmeyer 2011: 195). Die Aufwertung der Kulturgeschichte lässt sich zwar, wie Elisabeth Erdmann nachgewiesen hat, in dem Zeitraum zwischen den 1850er und 1880er Jahren auch in Schulgeschichtsbüchern für Knabenschulen feststellen (Erdmann 1982: 89f.). Als ideales Anschauungsmaterial bei der Vermittlung patriotischer Werte fällt sie bei Schulgeschichtsbüchern für höhere Mädchenschulen jedoch deutlicher ins Gewicht.

Die Betonung der jüngsten vaterländischen Geschichte und die Vermittlung gesellschaftlicher Werte anhand kulturhistorischer Beispiele folgten dabei den ministerialen Erlassen auf Landes- und Reichsebene seit den 1880er Jahren. So heißt es in den Bestimmungen über das Mädchenschulwesen, die Lehrerinnenbildung und die Lehrerinnenprüfungen der preußischen Kultusbehörde von 1894: »Der Unterricht erstrebt Stärkung und Vertiefung der Liebe zu Vaterland, Heimath und Herrscherhaus, Verständnis für das Leben der Gegenwart und die Aufgaben unseres Volkes [...]« (Ministerium 1894: 29). Die Furcht der Regierung vor einer Störung der gesellschaftlichen Ordnung spiegelt sich in den verschiedenen ministerialen Erlassen wider. So sollte der Geschichtsunterricht der Allerhöchsten Order Wilhelms II. vom 1. Mai 1889 zufolge zusammen mit dem Religions- und Deutschunterricht einen Beitrag dazu leisten, die Sozialisationsziele Gottesfurcht, Liebe zum Vaterland und Königstreue durchzusetzen (Schneider 1982: 134). Die von Wilhelm II. am 30. August 1889 genehmigten Vorschläge zur Ausführung des Allerhöchsten Befehls beziehen sich ausdrücklich neben den Gymnasien auch auf die Volks-, Mittel- und Mädchenschulen. Dadurch rückte die Neuere Geschichte ab den 1890er Jahren ins Zentrum der vermittelten Unterrichtsinhalte. Die damit einhergehende Abwertung der Alten Geschichte ist auch als Versuch zu verstehen, den Kosmopolitismus an den höheren Schulen zurückzudrängen, der im Verdacht stand, den Republikanismus zu befördern und das Nationale zu unterhöhlen. 


\section{ZUSAMMENFASSUNG}

In dem vergleichsweise kurzen Zeitraum zwischen dem 18. Jahrhundert und dem Beginn des 20. Jahrhunderts bildete sich eine Form der Geschichtsschreibung heraus, die sich vorrangig an ein weibliches Lesepublikum richtete. Ersten Befunden zufolge lassen sich dabei verschiedene Typen unterscheiden: Erstens erschienen in den Frauenzeitschriften, die sich seit dem späten 18. Jahrhundert herausgebildet hatten, neben Novellen, Fortsetzungsromanen, Gedichten und Andachten auch historisch-politische Artikel häufig unterhaltsamer Provenienz. Zweitens wandten sich Vertreter des Faches in speziellen Vortragsreihen, die häufig auch publiziert wurden, an ein weibliches Publikum, wobei sie geschlechtsspezifische didaktische und thematische Schwerpunkte setzten. Drittens entstanden im 19. Jahrhundert eigene Schulgeschichtsbücher für das höhere Mädchenschulwesen, die aufgrund der institutionellen Vorgaben die größte Verbreitung gefunden haben. Zeitlich waren diese am deutlichsten eingegrenzt, da ab ca. 1910 mit der Angleichung der höheren Bildungsanstalten für Jungen und Mädchen einheitliche Geschichtsschulbücher verwendet werden sollten.

Voraussetzung für die Verbreitung von Schulgeschichtsbüchern an Mädchenschulen war der Aufschwung des Mädchenschulwesens, der mit institutionellen Vorgaben von ministerialer Seite ab der Mitte des 19. Jahrhunderts einherging. Die Verwendung eines Lehrbuchs im Geschichtsunterricht wurde seit den 1850er Jahren auch an Mädchenschulen obligatorisch. Dennoch blieb die Zahl an eigenen Schulgeschichtsbüchern für Mädchen gering. Größte Verbreitung fanden Friedrich Nösselts Lehrbuch der Weltgeschichte für Töchterschulen und zum Privatunterricht heranwachsender Töchter von 1822 sowie Carl Wernickes Weltgeschichte für höhere Töchterschulen von 1849, die bis in die 1890er Jahre hinein vielfach aufgelegt wurden. Einen Wendepunkt stellten die Bestimmungen über das Mädchenschulwesen, die Lehrerinnenbildung und die Lehrerinnenprüfungen des preußischen Kultusministeriums vom 31. Mai 1894 dar, woraufhin sich die Produktion von Schulgeschichtsbüchern für Mädchenschulen deutlich erhöhte. Die Erfolge der Frauenbewegung und das Aufbrechen einer starren Geschlechteranthropologie drängte eine spezielle Darstellung der Geschichte für ein weibliches Publikum ab dem Beginn des 20. Jahrhunderts allerdings zurück.

Begründet wurde eine gesonderte Form der Geschichtsschreibung für Mädchen und junge Frauen durch die naturgegebenen Geschlechterunterschiede, die seit dem letzten Drittel des 18. Jahrhunderts festgeschrieben worden waren (Honegger 1991). Der Aufteilung der Sphären in Öffentlichkeit und Privatheit folgend, wurde das universalisierte Ideal der dreifachen Bestimmung der (bürgerli- 
chen) Frau als Gattin, Hausfrau und Mutter auf zurückliegende historische Epochen übertragen. Stärker als in den Geschichtsbüchern für Knabenschulen folgte die Darstellung dabei moralisch-sittlichen Mustern, wobei die Geschichte als Ort der Wertevermittlung angenommen wurde. Weibliche Tugenden wie Keuschheit, Treue, Sittlichkeit und Geduld hätten demzufolge auch in historischen Umbruchszeiten stabilisierend gewirkt. Bei der Begründung der Wertevermittlung für das »moralische Geschlecht« (Steinbrügge 1987) lösten im letzten Drittel des 19. Jahrhunderts zunehmend patriotisch-nationale Vorgaben die älteren christlich-sittlichen Argumentationsmuster ab.

Aus der zugrundeliegenden Geschlechteranthropologie ergaben sich maßgebliche Unterschiede bei der Darstellung von Geschichte im Knaben- und Mädchenunterricht. Mit der größeren Anschaulichkeit, der Emotionalisierung und Personalisierung der Geschichte sowie der Reduktion von Komplexität wurden für den Mädchenunterricht Darstellungsformen gefordert, die sich auch in der populären Geschichtsliteratur finden. Thematisch standen Kultur- und Sittengeschichte im Mittelpunkt. Bei der Politik-, Militär- und Verfassungsgeschichte sei ein grober Überblick ausreichend. Die Geschichte der Frauen, die dem biographischen Prinzip folgend am historischen Beispiel vermittelt werden sollte, kam als weiterer thematischer Schwerpunkt hinzu.

Trotz der Unterschiede in den Darstellungsformen sind die Parallelen in den Schulgeschichtsbüchern für den Knaben- und Mädchenunterricht nicht zu übersehen. Die Vermittlung nationaler Werte war den ministerialen Vorgaben zufolge auch an den Knabenschulen der Hauptzweck des Unterrichts. Die Erzählung als zentrale Darstellungsform setzte sich in der Historiographiegeschichte des 19. Jahrhunderts insgesamt durch. Die führenden Pädagogen des 19. Jahrhunderts waren sich einig, für Mädchen und junge Frauen eine spezielle Form der Geschichtsschreibung zu fordern. Inwieweit dies jedoch in den Darstellungsteilen tatsächlich umgesetzt wurde, gilt es weiter zu untersuchen.

\section{LITERATUR}

Biedermann, Karl (1856): Frauen-Brevier: Kulturgeschichtliche Vorlesungen, Leipzig: Weber.

Epple, Angelika (2003): Empfindsame Geschichtsschreibung: Eine Geschlechtergeschichte der Historiographie zwischen Aufklärung und Historismus, Köln [u.a.]: Böhlau.

Epple, Angelika (2011): »Questioning the Canon: Popular Historiography by Women in Britain and Germany (1750-1850)«. In: Sylvia Paletschek (Hg.), 
Popular Historiogrpahies in the 19th and 20th Centuries: Cultural Meanings, Social Practices, Oxford [u.a.]: Berghahn Books, S. 21-33.

Erdmann, Elisabeth (1982): »Tendenzen und Neuansätze in Geschichtsdidaktik und Geschichtsunterricht nach 1848 bis in die Mitte der achtziger Jahre des 19. Jahrhunderts«. In: Klaus Bergmann/Gerhard Schneider (Hg.), Gesellschaft, Staat, Geschichtsunterricht, Düsseldorf: Pädagogischer Verlag Schwann, S. 77-103.

Honegger, Claudia (1991): Die Ordnung der Geschlechter: Die Wissenschaften vom Menschen und das Weib (1750-1850), Frankfurt am Main [u.a.]: Campus-Verlag.

Jacobmeyer, Wolfgang (2011): Das deutsche Schulgeschichtsbuch 1700-1945:

Die erste Epoche seiner Gattungsgeschichte im Spiegel der Vorworte, Berlin [u.a.]: LIT-Verlag.

Jäger, Georg/Jörg Schönert (1980): »Die Leihbibliothek als literarische Institution im 18. und 19. Jahrhundert - ein Problemaufriß«. In: Dies. (Hg.), Die Leihbibliothek als Institution des literarischen Lebens im 18. und 19. Jahrhundert, Hamburg: Hauswedell 1980, S. 7-60.

Kraul, Margret (1991): »Höhere Mädchenschulen«. In: Christa Berg (Hg.); Handbuch der deutschen Bildungsgeschichte. Band 4, München: Beck, S. 279-303.

Küpper, Erika (1991): »Die höheren Mädchenschulen«. In: Karl-Ernst Jeismann/ Peter Lundgreen (Hg.), Handbuch der deutschen Bildungsgeschichte. Band 3, München: Beck, S. 180-191.

Langenbucher, Wolfgang R. (1971): »Das Publikum im literarischen Leben des 19. Jahrhunderts«. In: Marion Beaujean (Hg.), Der Leser als Teil des literarischen Lebens, Bonn: Bouvier, S. 52-84.

Ministerium der Geistlichen, Unterrichts- und Medizinalangelegenheiten (1894): Bestimmungen über das Mädchenschulwesen, die Lehrerinnenbildung und die Lehrerinnenprüfung vom 31. Mai 1894, Berlin: Wilhelm Hertz.

Nissen, Martin (2009): Populäre Geschichtsschreibung: Historiker, Verleger und die deutsche Öffentlichkeit (1848-1900), Köln [u.a.]: Böhlau.

Nösselt, Friedrich (1836): Lehrbuch der Weltgeschichte für Töchterschulen und zum Privatunterricht heranwachsender Töchter. Teil 1-3, 5. Aufl., Breslau: Max.

Oeser, Chr. [d. i. Tobias Gottfried Schröer] (1843): Weltgeschichte für Töchterschulen und zum Privatunterricht. Teil 1-3, 2. Aufl., Leipzig: Wilhelm Einhorn.

Paletschek, Sylvia (2006): »Ermentrude und ihre Schwestern: Die ersten habilitierten Historikerinnen in Deutschland«. In: Henning Albrecht et al. (Hg.), 
Politische Gesellschaftsgeschichte im 19. und 20. Jahrhundert, Hamburg: Krämer, S. 175-187.

Rohlfes, Joachim (1982): »Geschichtsunterricht in Deutschland von der frühen

Neuzeit bis zum Ende der Aufklärung «. In: Klaus Bergmann/Gerhard Schneider (Hg.), Gesellschaft, Staat, Geschichtsunterricht, Düsseldorf: Pädagogischer Verlag Schwann, S. 11-43.

Schneider, Gerhard (1982): »Der Geschichtsunterricht in der Ära Wilhelms II. (vornehmenlich in Preußen)«. In: Klaus Bergmann/Gerhard Schneider (Hg.), Gesellschaft, Staat, Geschichtsunterricht, Düsseldorf: Pädagogischer Verlag Schwann, S. 132-189.

Schneider, Gerhard (1988): »Geschichtsdidaktik und Geschichtsunterricht am Ende des Kaiserreichs (vorwiegend in Preußen)«. In: Paul Leidinger (Hg.), Geschichtsunterricht und Geschichtsdidaktik, Stuttgart: Ernst Klett Verlag, S. 54-67.

Schneider, Gerhard (1997): »Geschichtsunterricht als Institution«. In: Klaus Bergmann et al. (Hg.), Handbuch der Geschichtsdidaktik, Seelze-Velber: Kallmeyer, S. 495-509.

Smith, Bonnie G. (1998): The Gender of History: Men, Women, and Historical Practice, Cambridge, Mass. [u.a.]: Harvard University Press.

Steinbrügge, Lieselotte (1987): Das moralische Geschlecht: Theorien und literarische Entwürfe über die Natur der Frau in der französischen Aufklärung, Weinheim [u.a.]: Beltz.

Weckel, Ulrike (1998): Zwischen Häuslichkeit und Öffentlichkeit: Die ersten deutschen Frauenzeitschriften im späten 18. Jahrhundert und ihr Publikum, Tübingen: Niemeyer.

Wittmann, Reinhard (1999): Geschichte des deutschen Buchhandels, 2. Aufl., München: Beck. 
Elisabeth Cheauré, Sylvia Paletschek and Nina Reusch - 9783839423738

Downloaded from PubFactory at 08/15/2016 01:34:22PM

via Universität Heidelberg 